\title{
Role of mixed ligand Pd(II) and Pt(II)complexes in photooxygenation reaction
}

\author{
V. V. Sawant ${ }^{1}$, S.R.Patil ${ }^{2} \& S$. T. Shukla ${ }^{1^{*}}$ \\ 1.Department of Chemistry, Y. C. Institute of Science, Satara, M.S. (India) \\ 2.Department of Chemistry, Shivaji University, Kolhapur,M.S.(India)
}

\begin{abstract}
Mixed ligand complexes of the formula [M (phen) bdt] where $M=P d$ (II) or Pt(II), phen $=1,10$ phenanthroline and bdt =1,2-benzenedithiolate have been synthesized and studied as photosensitizers of singlet molecular oxygen $\left({ }^{b} \mathrm{O}_{2}\right.$ ) production. It was observed that, Pt (II) complex shows a better photosensitizing ability as compared to its Pd (II) analogue. However, both Pd (II) and Pt (II) complexes act as chemical quenchers of ${ }^{I} O_{2}$, resulting in their photooxidation shown by decay of the respective complex. ThusPt (II) complex undergoes selfsensitizedphotooxidation by ${ }^{I} \mathrm{O}_{2}$. The solvent dependence of this reaction is undertaken by performing the experiment using pure DMF(dimethyl formamide) and $10 \% \mathrm{H}_{2} \mathrm{O}$ in DMF as solvent. The comparison of rate of decay of the Pt(II) and Pd(II) complexes show enhancement of the reaction in presence of water. The decay of the complex was monitored by recording the difference absorption spectra of the complex in the visible region after definite irradiation times. Involvement of ${ }^{I} \mathrm{O}_{2}$ is further supported by quenching studies of this reaction using $\mathrm{NaN}_{3}$ as physical quencher of ${ }^{1} \mathrm{O}_{2}$.
\end{abstract}

Key words: chemical quenching,photooxygenation reaction, photosensitizer, photooxidation,singlet oxygen

\section{Introduction:}

Photochemistry plays an important role in producing chemical compounds or bringing them into reaction. The development of chelating ligands and their transition metal complexes has been of increasing interest. Square-planar Pt(II), Pd(II), and $\mathrm{Au}(\mathrm{III})$ terpyridine complexes show their potential as antitumor and antiprotozoal agents. Photosensitization is an important photochemical reaction involving generation and quenching of singlet molecular oxygen $\left({ }^{1} \mathrm{O}_{2}\right)$, which is one of the most active intermediates in chemical and biochemical reactions[1].

The study of singlet molecular oxygen production and reactivity has emerged as a rich and diverse area with implications in various fields. It has a characteristic chemistry that sets it apart from the triplet ground state of molecular oxygen $\left({ }^{3} \mathrm{O}_{2}\right)$ and is important in fields that range from atmospheric chemistry and material science to biology and medicine[2]. It is found to play major role in photodynamic effect. Application of this effect to blood sterilization, cancer therapy, and insecticides, pesticides is of increasing importance. The literature has offered new data related to its application in wastewater treatment[3]. Therefore study of generation and quenching of singlet oxygen becomes important.It has been demonstrated that $\operatorname{Pt}(\mathrm{II})$ and $\mathrm{Pd}(\mathrm{II})$ diimine complexes sensitize the formation of singlet oxygen $\left({ }^{1} \mathrm{O}_{2}\right)$ from ground state molecular oxygen $\left({ }^{3} \mathrm{O}_{2}\right)$ Photosensitizing ability of $\mathrm{Ru}$ (II) complexes has also been studied. Ni(II) complexes have been reported to be quenchers of singlet oxygen[4-6].

[ Pd(phen)DMT] has been reported as acceptor (chemical quencher) of singlet molecular oxygen whereas the analogous Pt(II) complexes have shown to undergo self-sensitizedphotooxidation [7].

\subsection{Materials and equipments:}

\section{Experimental:}

The chemicals used for the present study, potassium tetrachloroplatinate $\left[\mathrm{K}_{2} \mathrm{PtCl}_{4}\right]$ (sd-Fine), $\mathrm{PdCl}_{2}$ (Sigma Aldrich), 1,2-benzenedithiol(bdt) (Aldrich) \& 1,10 phenanthroline (phen), Sodium azide $\left(\mathrm{NaN}_{3}\right)$, Hematoporphyrin, Dimethylformamide (DMF), Dimethylsulphoxide (DMSO), Chloroform and Acetone were purchased from SISCO. Microprocessor photocolorimeter (Esico model-1313) and Double Beam UV/VIS Spectrophotometer (Systronics-Model AU- 2700/2701)was used to measure absorbance before and after irradiation. Tungsten-Halogen lamp $(300 \mathrm{~W})$ was used for irradiation purpose. A transparent colourless acrylic box $(21.5 \mathrm{~cm} \times 11.5 \mathrm{~cm} \times 11.5 \mathrm{~cm})$ was used as heat filter to prevent the sample solution from getting heated.

\subsection{Synthetic work:}

$[\mathrm{M}(\mathrm{phen}) \mathrm{bdt}]$ : Mixed ligand complexes of the type [M(phen)bdt] where $\mathrm{M}=\mathrm{Pd}(\mathrm{II})$ or $\mathrm{Pt}(\mathrm{II})$ have been synthesized by the procedure reported in the literature $[6,8]$. 


\subsection{General Irradiation Procedure:}

For irradiation, a wooden box $(75 \mathrm{~cm} \times 25 \mathrm{~cm} \times 19 \mathrm{~cm}$ ) was used as a dark chamber. At one end of this wooden box, the light source was fitted. The acrylic box was placed at a fixed distance $(15 \mathrm{~cm})$ from the source. The solution to be photolyzed was transferred into a cuvette having $1 \mathrm{~cm}$ path length and was placed at the other side of the acrylic box ( at a distance of $6 \mathrm{~cm}$ ) for irradiation. Absorbance of this solution was measured before and after irradiation.

\subsection{Photochemical Studies:}

A 0.2mM solution of [M (phen) ( bdt)] in DMF was bubbled with molecular oxygen for 20 minutes. It was irradiated for different time intervals and absorbance of the solution was measured as a function of time. Taking into account the difference in behaviour, separate experiments were performed for $\mathrm{Pd}(\mathrm{II})$ and $\mathrm{Pt}(\mathrm{II})$ complexes.

\subsection{1[Pt(phen)(bdt)] :}

Photolysis of [Pt(phen)(bdt)] was performed with and without sodium azide, a well known Physical quencher of ${ }^{1} \mathrm{O}_{2}$.For this, pilot experiments were performed with different concentrations of the complex and $\mathrm{NaN}_{3}$ to select optimum concentration range. Oxygen saturated solution of [Pt(phen)bdt] $(0.2 \mathrm{mM})$ and different concentrations of $\mathrm{NaN}_{3}(0,0.05 \mathrm{mM}$ and $0.1 \mathrm{mM})$ was irradiated and decay of the complex was monitored by noting the absorbance after definite irradiation times. Photolysis of molecular nitrogen saturated solution of the complex was also studied.

\subsection{2 [ Pd(phen)(bdt)] :}

After a series of pilot experiments the optimum concentration of solution to be photolyzed was decided. Photolysis of [Pd(phen)bdt] $(0.5 \mathrm{mM})$,Hematoporphyrin $(0.08 \mathrm{mM})$ and sodium azide $(0.05 \mathrm{mM})$ was carried out . A parallel experiment was also performed in absence of sodium azide for comparison. Pure oxygen gas was bubbled through the solutions for 15 minutes before irradiation. Similar experiment was performed after passing molecular nitrogen through the experimental solution.

\subsubsection{Solvent Effect:}

Photolysis of the complex has been performed using $10 \%$ aqueous DMF to study the effect of solvent. The reaction was monitored by recording difference absorption spectra after definite time intervals.

\section{Results and Discussion:}

The complexes were characterized by IR and NMR. The spectra obtained are in good agreement with those reported in the literature[9-11]. The [M(phen)bdt]complexes show five absorption bands. The lowest triplet state ${ }^{3} \mathrm{~S}_{1}$ of the longest wavelength is photochemically important as shown earlier in [ $\left.\mathrm{Pd}(\mathrm{bpy})(\mathrm{dmt})\right]$.

\subsection{Photochemical studies:}

\subsection{1 [Pd(phen)bdt] as an acceptor of ${ }^{1} \mathrm{O}_{2}$ :}

Absorbance of Oxygen saturated solution containing $0.5 \mathrm{mM} \quad[\operatorname{Pd}(\mathrm{phen}) \mathrm{bdt}]$ and $0.08 \mathrm{mM}$ Hematoporphyrin at $500 \mathrm{~nm}$ was found to decrease which may be due to the decay of [ $\operatorname{Pd}(\mathrm{phen}) \mathrm{bdt}]$ complex. However, when the mixture was irradiated in presence of pure molecular nitrogen, this decay was not observed. Also, there was no decrease in absorbance if the solution was kept in dark. There was $5 \%$ decrease in absorbance when oxygen saturated solution of $[\operatorname{Pd}(\mathrm{phen}) \mathrm{bdt}]$ was irradiated for 4 hours. Therefore it can be concluded that the decrease in absorbance is due to the combined action of the complex [M(phen)bdt], Hematoporphyrin, light and molecular oxygen. Hematoporphyrin is a well-known sensitizer of ${ }^{1} \mathrm{O}_{2}$. Therefore this decay might involve singlet molecular oxygen(fig.1). Involvement of singlet oxygen is proved by quenching of this decay in presence of sodium azide.

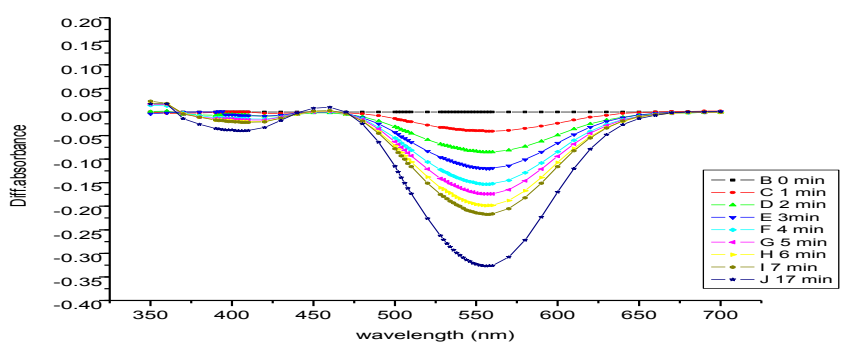

Fig 1: Decay of the complex on irradiation in presence of hematoporphyrin 
From the above results, it can be noted that $\mathrm{Pd}(\mathrm{II})$ complex under study acts as chemical quencher of ${ }^{1} \mathrm{O}_{2}$. This can be explained by the following mechanism[12]-

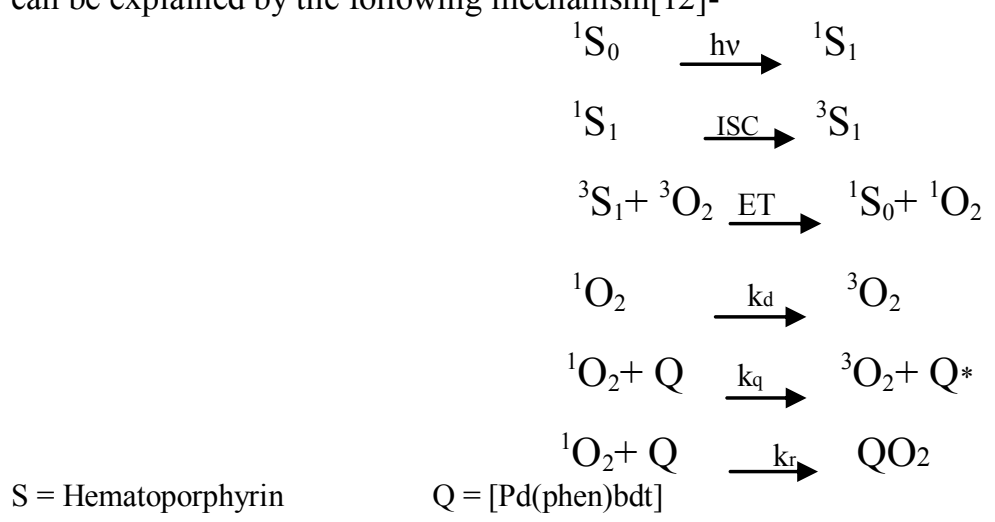

\subsubsection{Self-sensitized photooxidation of $[\operatorname{Pt}($ phen)bdt]:}

Oxygen saturated solution of [ $\mathrm{Pt}(\mathrm{phen}) \mathrm{bdt}](0.2 \mathrm{mM})$ in DMF was irradiated withvisible radiation . The absorbance was noted at different times of irradiation to study thephotolysis. The photolysis results in decay of the complex which is shown by decrease inabsorbance of the solution. The change in absorbance corresponds to the amount reacted(x) and initial absorbance is taken as "a". A graph is plotted between (x/a-x) of the complex vs. irradiation time (Fig:2.1 and 2.2).

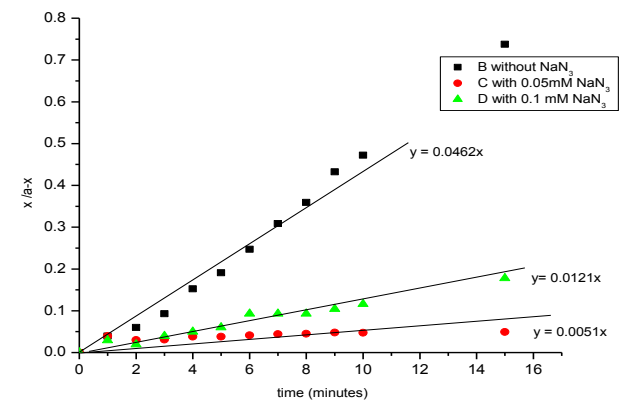

Fig.2.1 plot of (x/a-x) of the complex[Pt(phen)(bdt)] and different concentrations of sodium azide against time

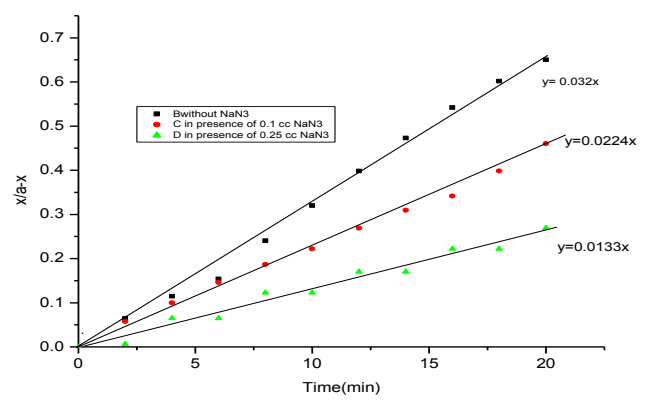

Fig.2.2:plotof ( $\mathrm{x} / \mathrm{a}-\mathrm{x})$ of the complex $[\mathrm{Pd}(\mathrm{phen})(\mathrm{bdt})]$ and different concentrations of sodium azide against time

Linear nature of the plot indicates that the decay of [M(phen)bdt] follows second order kinetics. This is also confirmed by log-log plot .This photolysis was further studied in presence of known ${ }^{1} \mathrm{O}_{2}$ quencher such as sodium azide $\left(\mathrm{NaN}_{3}\right)$. It was found that the decay of the complex was reduced when $\mathrm{NaN}_{3}$ was added to [Pt(phen)bdt] and then irradiated. Oxygen saturated solution of [Pt(phen)bdt] $(0.2 \mathrm{mM})$ and $\mathrm{NaN}_{3}(0.1 \mathrm{mM})$ was irradiated and decay of the complex was monitored by noting the absorbance after definite irradiation times. It can be seen from Fig. 1 that when concentration of $\mathrm{NaN}_{3}$ is increased, decay of the complex is reduced. A comparison of the slopes of lines confirms this trend. A remarkable change in the color of the oxygen saturated solution of [Pt(phen)bdt] in DMF was observed before and after irradiation. Reddish purple color of the solution before irradiation changed to pale yellow after irradiation for thirty minutes. This may be due to a chemical reaction between $[\mathrm{Pt}(\mathrm{phen}) \mathrm{bdt}]$ and ${ }^{1} \mathrm{O}_{2}$ produced during photolysis. Quenching of this reaction in presence of $\mathrm{NaN}_{3}$, indicates the involvement of singlet oxygen. This was further confirmed by the fact that there was no decrease in absorbance on photolysis in presence of pure molecular nitrogen. Also, if this solution was kept in dark, no decrease in absorbance was observed. Thus it can be concluded that the above decay requires combined action of [Pt(phen)bdt], light and oxygen. This indicates that the complex acts as photosensitizer as well as acceptor of singlet oxygen. The process is thus referred to as self sensitized photooxidation. The mechanism proposed for this reaction is similar to that explained earlier for [ $\mathrm{Pt}(\mathrm{phen}) \mathrm{DMT}]$ and [Pt(phen)TSA] [12].

Mixed ligand complexes containing 1,10-phenanthroline as one of the ligands and catechol or tertiary butyl catechol as the other have also been reported to be stable to light irradiation for 5-6 hours. This confirms that it is not the phenanthroline moiety in [Pt(phen)bdt]which is attacked during photooxidation[7]. Thus it can be concluded that it must be the 1,2- benzenedithiol moiety which is attacked by ${ }^{1} \mathrm{O}_{2}$. This is supported by the literature which reveals the interaction of thioethers with ${ }^{1} \mathrm{O}_{2}$ to give sulphoxides. Also it has been shown that [Pt(bpy)bdt] where bpy $=2,2^{\prime}$ bipyridine can quench ${ }^{1} \mathrm{O}_{2}$ to form sulphonated products[8].

The ${ }^{1} \mathrm{O}_{2}$ production by the $[\mathrm{M}(\mathrm{phen}) \mathrm{bdt}]$ complex depends on the ligand field splitting by the ligands attached to the metal. But in this case, as both ligands are same, we consider the metal. In Pt(II), the ligand field 
increases by $30 \%$ to $50 \%$ from the corresponding Pd(II) complexes. Therefore the ${ }^{3} \mathrm{~A}_{2}$ state has much higher energy with respect to ${ }^{3} \mathrm{~S}_{1}$. Thus $\mathrm{Pt}(\mathrm{II})$ complexes are better sensitizers. However in $[\mathrm{Pd}(\mathrm{phen})$ bdt],they may have the ${ }^{3} \mathrm{~A}_{2}$ state just below the ${ }^{3} \mathrm{~S}_{1}$ which provides pathway of radiationless decay resulting in its decreased photosensitizing ability.

\subsubsection{Photooxygenation of complexes in mixed solvent:}

Review of literature reveals that reaction of metal thiolato complex with singlet oxygen can lead to several products [9-11] as -

i. Formation of sulfenate at one of the sulfur sites only.

ii. Formation of a bis-sulfenate.

iii. Formation of mono or bissulfinate.

iv. Formation of a mixed sulfenato/sulfinato complex.

In Co(III) thiolato complexes it has been reported earlier that protic solvents decrease the susceptibility of the thiolate ligand toward attack by singlet oxygen, but greatly increase the conversion of the peroxidic intermediate to the sulfenato product[13,14].
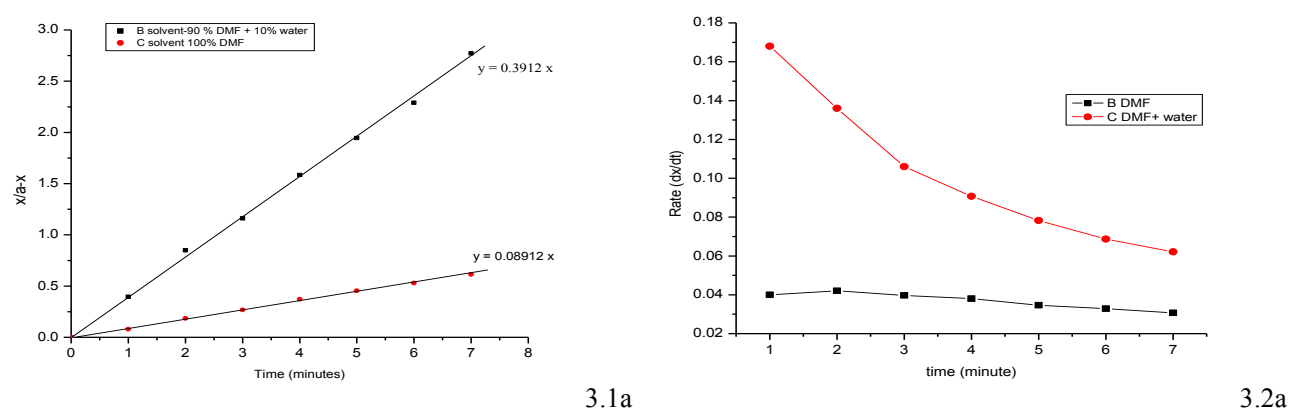

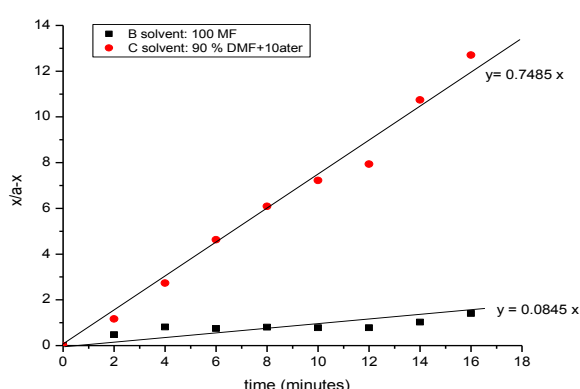

Fig 3.1a \&b: Plot of (x/a-x) against time
$3.1 \mathrm{a}$

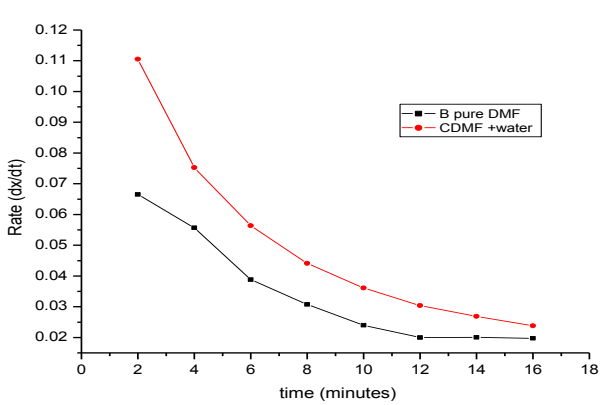

$3.1 \mathrm{~b}$

Fig $3.2 \mathrm{a} \& \mathrm{~b}$ : Plot of rate against time
$3.2 b$

Fig 3.1 a \&b: the second order plots of [Pt(phen)(bdt)] and [Pd(phen)(bdt)] . The comparison of slopes of the lines suggests the enhancement in reaction in presence of aqueous (10\%water in DMF)solvent.Fig $3.2 \mathrm{a} \& \mathrm{~b}$ : The plot of rate $(\mathrm{dx} / \mathrm{dt})$ against time shows increase in rate of photolysisof the complexes. Higher rate of reaction may be due to stabilisation of reaction intermediate in presence of water(protic solvent) [14].

\section{Conclusion:}

Comparative study of $[\mathrm{M}(\mathrm{phen})(\mathrm{bdt})]$ shows that $[\mathrm{Pt}(\mathrm{phen})(\mathrm{bdt})]$ acts as photosensitizer as well as acceptor (chemical quencher) of ${ }^{1} \mathrm{O}_{2}$ whereas [Pd(phen)(bdt)] although not a photosensitizer, acts as chemical quencher of ${ }^{1} \mathrm{O}_{2}$. The observations suggest an increase in the reaction rate when the solvent DMF contains $10 \%$ water.This may be due to the stabilization of the intermediate in presence of water.It has been reported recently that mixed ligand Pd(II) complexes exhibit significant biological activity[15]. Thus, in future, these complexes can be tested for their biological activity.

\section{Acknowledgement:}

This research was sponsored by the University grant Commission. Their support isgratefully acknowledged. Special thanks are due to Dr.ArvindBurungale, Principal,Y.C. Institute of Science Satara, for his support and encouragement. 


\section{References:}

[1]. Eryazici I, Moorefield Charles N \&Newkome George R, Square-Planar Pd(II), Pt(II), and Au(III) Terpyridine Complexes: Their Syntheses, Physical Properties, Supramolecular Constructs, and Biomedical Activities,ChemicalReviews, vol. 108, No. 6,2008, 1834-1895

[2]. Peter ROgilby, Singlet oxygen: there is indeed something new under the sun, Chem.SocRev , 39(2010) 3181-3209

[3]. De Rosa Maria C, R J Crutchley, Photosensitized singlet oxygen and its application,Co-ordination Chemistry Reviews, 233234(2002)351-371

[4]. V.AnbalganandT S Srivastava, Investigation of ${ }^{1} \mathrm{O}_{2}$ generation by water soluble $\alpha$-diiminecomplexes of platinum(II) and palladium(II) with 3,4-dihydroxybenzoic acid as photosensitizers, J photochemphotobiol A:Chemistry, 66(1992) 345-353

[5]. C.A. Gruperhaus, Oxygen Capture by Sulfur in Nickel Thioates, Acc. Chem. Res., 31(1998) 451-459

[6]. T.MathewsCocker and Robert E. Bachman, Photochemical and Chemical oxidation of $\alpha$-Dimine-Dithiolene Metal Complexes:Insightinto the Role of the Metal Atom, Inorg.Chem,40,.2001,.1550-1556

[7]. S SKamath, S Shuklaand T S Srivastava, Mixed ligand palladium(II) and platinum(II) complexes-photosensitizers of ${ }^{1} \mathrm{O}_{2}$ production and chemical quenchers of ${ }^{1} \mathrm{O}_{2}$, Bull.Chem.Soc.Jpn, 64 ,1991, 1351-1359

[8]. S D Cummings, R Eisenberg, Tuning the excited Propertiseof Platinum (II) diimineDithiolate complexes,J. Am Chem.Soc, 118, 1996, 1949-1960

[9]. C.Makedonas, C AMitsopoulou, F J Lahoj, A J Balana, Synthesis Characterisation and Crystal Structure of The Pd(phen)(bdt) complex.A DFT and TDDFT Study of Its Ground Electronic and Excited states compared to those of Analogous complexes, InorgChem, 42, 2003, 8853-8865

[10]. W B Connick, H B Gray, Photooxidation of Platinum (II) DiimineDithiolates, J Am ChemSoc, 119, 1997, 11620-11627

[11]. Edward L. Clennan,New Mechanistic and Synthetic Aspects of Singlet Oxygen Chemistry, Tetrahedron ,56, 2000, $9151 \pm 9179$

[12]. S. Shukla, S SKamath,T S Srivastava, Self-Sensitized Photo-Oxidation Of Platinum(H) Complexes Of 1,10-Phenanthroline With 3,4- Dimercaptotoluene And Thiosalicylic Acid By ${ }^{1} 0_{2}$,J photochemphotobiol, A:Chemistry,50, 1989, 199-207

[13]. B.Hernandez ,Y.Wang,D.Zhang and M.Selke,Photooxidation of Co complexes in protic and aprotic solvents,Chem.Commun.,2006,997-999

[14]. D.Zang,B.Hernandez and M.Selke,Photooxidation of metal bound thiolates:reactivity of sulphur containing peroxidic intermediates, J.Sulphur Chem., 29(3), 2008, 377-388.

[15]. EnJunGao, Feng Guan, XiaNanGao, Ming Chang Zhu, Lei Liu, ChuanShengWang, WanZhong Zhang, YaGuang Sun, Novel palladium(II) complexes containing a sulphur ligands:structure and biologicalactivity on Hella cell, J BiolInorgChem, 17 (2012),263-274 